\title{
Linx
}

Revue des linguistes de l'université Paris X Nanterre

$60 \mid 2009$

Nonne scripta manent?

\section{À la mémoire de Jacques Anis}

Michel Arrivé

\section{(2) OpenEdition}

Journals

Édition électronique

URL : http://journals.openedition.org/linx/663

DOI : 10.4000/linx.663

ISSN : 2118-9692

Éditeur

Presses universitaires de Paris Nanterre

Édition imprimée

Date de publication : 1 mai 2009

Pagination : 7-15

ISSN : 0246-8743

\section{Référence électronique}

Michel Arrivé, «À la mémoire de Jacques Anis », Linx [En ligne], 60 | 2009, mis en ligne le 24 février 2017, consulté le 21 septembre 2020. URL : http://journals.openedition.org/linx/663 ; DOI : https:// doi.org/10.4000/linx.663 


\section{À la mémoire de Jacques Anis}

Michel Arrivé

Jacques Anis est mort, à l'âge de cinquante-deux ans, le 16 décembre 2005, emporté par la généralisation de la myopathie dont il souffrait depuis sa naissance. Elle avait semblé donner, pendant son adolescence et jusque dans ses premières années d'adulte, quelques signes de rémission, qui avaient même pu faire penser à une guérison. Espoir bientôt déçu : la maladie reprit son cours.

Est-ce la perspective incessamment présente d'une mort précoce qui a fait de Jacques Anis un homme constamment pressé par le temps? Je ne sais : il ne parlait guère des soucis que lui causait sa maladie. Ce que je sais, ce que tout le monde sait, c'est qu'il a essayé d'accélérer le rythme de sa vie, et y a souvent réussi. Les retards qu'il a subis lui ont été imposés par des forces - souvent des volontés - extérieures.

Les études de Jacques Anis ont été particulièrement précoces et brillantes. Venu de la khâgne du lycée Condorcet - il en gardait d'excellents souvenirs - il a été admis à l'ENS de la rue d'Ulm en 1972, à dix-neuf ans. Deux ans plus tard, en 1974, il est reçu à l'agrégation de lettres classiques. Il a vingt et un ans, et c'est un record de précocité agrégative qui, à ma connaissance, n'est pas encore battu : en quinze ans de présence aux jurys de deux agrégations littéraires, je l'ai vu deux ou trois fois égalé, mais jamais surpassé.

Après l'agrégation, Jacques Anis reste pendant deux ans à l'École Normale, puis est nommé pour trois ans attaché de recherches au CNRS, spécifiquement à la Fondation Thiers. Je ne sais plus si c'est dès octobre 1974 ou en janvier 1975 - trente ans, quoi qu'il en soit, avant sa mort - que, tout jeune agrégé, il est venu me voir, à Nanterre, pour envisager de déposer un sujet de thèse de $3^{\text {ème }}$ cycle. Il hésitait encore entre deux centres d'intérêt. Le lexique de la lutte des classes dans la littérature du XIX ${ }^{\mathrm{e}}$ siècle l'attirait fortement. Après avoir hésité quelque temps, nous avons pris une 
direction toute différente: nous avons pensé à examiner les problèmes de ce qu'il appellera, un peu plus tard, d'un très beau mot-valise, la "vilisibilité » du texte poétique. Il s'agissait de poser les méthodes de la description du texte poétique envisagé dans la matérialité de sa réalisation graphique dans l'espace textuel. Après avoir pensé au "Coup de dés », Anis a finalement choisi pour corpus les sonnets de Mallarmé réunis sous le titre «Plusieurs sonnets » et Les montagnes rocheuses de Michel Butor.

Jacques Anis termine sa thèse en trois ans : il est docteur en 1978, à vingt-cinq ans. Nouveau record de précocité, sans doute lui aussi difficile à battre, voire à égaler. La thèse est longue - 313 pages, très serrées - et, inévitablement, très austère. Elle n'a pas été publiée. Mais Anis a fait paraitre, dans les années 80 , plusieurs articles dans la mouvance de sa thèse. J'ai déjà cité "La vilisibilité du texte poétique », dans le numéro de Langue française qu'il a dirigé sur "Le signifiant graphique ». J'ajoute un très beau texte de LINX intitulé, de façon rimbaldienne, mais avec une légère déviation, apparemment atténuative, « Littéralement et dans plusieurs sens ».

Dès l'époque de sa thèse, Anis, on le voit clairement dans certains passages, commence à penser à ce qui va l'occuper pendant plusieurs années : le projet de construction d'une "graphématique autonome», c'est-à-dire d'une théorie de la manifestation écrite de la langue élaborée en dehors de toute relation avec la manifestation orale. C'est ce projet qui l'occupe, à temps plein, dès la fin des années 80. Il donnera lieu, en 1988, à la publication du livre intitulé L'écriture, théories et descriptions (avec la collaboration de Jean-Louis Chiss et Christian Puech).

La graphématique continue à occuper une place centrale dans les préoccupations de Jacques Anis jusqu'en 1990. C'est cette année-là qu'il soutient son Habilitation à Diriger des Recherches : l'un des cinq gros volumes qui constituent son dossier, le plus compact des cinq, est consacré à la graphématique. Il faut en ce point rappeler la nouveauté, à l'époque, de ce type de recherche. Et l'opposition virulente qu'elle détermine chez la plupart des spécialistes de l'écriture et de l'orthographe. Pour le jury de son HDR, j'avais fait appel à Nina Catach, en raison de sa compétence, généralement reconnue, dans le domaine de l'écriture. Je ne soupçonnais pas à quel point Nina Catach, qui était dans le privé, la personne la plus amène et la plus bienveillante du monde, était, sur le plan théorique, une sorte d'ogresse passionnée et cruelle. La «graphématique autonome» de Jacques Anis la précipitait dans des accès de fureur incontrôlable. André Eskénazi, qui présidait, avec sa pondération habituelle, la soutenance, ne parvenait à la calmer provisoirement que par de solennelles objurgations en alexandrins. Et sa rage mal éteinte s'est encore manifestée dans certains passages, à mon sens injustes, du rapport de soutenance.

En même temps qu'il travaillait, Anis progressait dans sa carrière. D'une façon moins rapide qu'il ne l'espérait : il a longtemps enseigné dans différents lycées avant d'être, en 1987, nommé Maître de conférences à Nanterre, neuf ans après la soutenance de sa thèse. Et il a dû attendre 1999, de nouveau neuf ans après la soutenance de son HDR, pour obtenir le poste de professeur qu'il méritait depuis longtemps. Il n'a atteint la première classe que peu de mois avant sa mort. Je le dirai d'un mot: l'excellence de ses travaux, le prestige de ses titres - notamment sa double qualité de normalien et d'agrégé - ont été, dans sa carrière, bien plutôt un handicap qu'un secours. Je sais qu'il en a souffert. 
L'écriture, dans tous ses états, dans tous les sens que peut prendre le beau mot d'écriture : c'est là l'objet central du travail d'Anis. Il participe activement aux travaux de l'ITEM, et publie plusieurs articles remarqués sur l'« avant-texte », à propos, notamment, de Ponge et de Valéry.

Déjà perceptible dans les travaux de graphématique, l'intérêt d'Anis pour les mutations du lire-écrire déterminées par la généralisation des techniques dérivées de l'informatique se précise et s'accentue dans les années 90 . Ses recherches finiront par produire ce qu'il appelle, dans un demi-sourire, son «grand œuvre ». C'est en effet un gros livre, 290 pages très compactes, publié en 1999 sous le titre interrogatif : Texte et ordinateur: l'écriture réinventée ? L'intérêt passionné, je crois ne pas exagérer, que Jacques Anis porte à ces problèmes se manifestera encore par de nombreux articles et par deux ouvrages réalisés sous sa direction. D'abord Internet, communication et langue française, en 1999, puis un petit livre très amusant, Parlę-vous texto ? Anis y révèle une autre face de son talent d'écriture : l'enjouement et la drôlerie.

Je n'ajouterai que quelques mots à ces brefs éléments de biographie. Pendant les trente années que je l'ai côtoyé, je voyais, objectivement, la maladie de Jacques Anis évoluer. Le normalien de 1972 enfourchait gaillardement son Vélosolex pour parcourir le quartier Latin. Le jeune agrégé des années 75 marchait presque normalement, et conduisait sa voiture. Le maitre de conférences de 1987 ne quittait déjà plus son fauteuil électrique. Et on sait de quelle façon la maladie a évolué depuis ce temps. Je crois n'être pas le seul à l'avoir éprouvé : Anis réussissait à faire oublier son handicap. Quand on l'entendait intervenir, dans une Assemblée générale du département, ou dans telle autre occasion, colloque ou soutenance de thèse, on oubliait le fauteuil électrique : on ne pensait plus qu'à la réflexion du chercheur ou du professeur.

Jacques Anis, j'en suis certain, se serait amusé d'apprendre que les Mélanges que, dès sa disparition, quelques-uns d'entre nous ont fait le projet d'offrir à sa mémoire, ont pris tant de temps pour parvenir enfin à la publication. Diverses circonstances indépendantes de la volonté des auteurs en sont responsables. Mais je gage qu'il aurait apprécié la composition de l'ouvrage: ce sont ses amis, et eux seuls, qui y ont contribué. Ils ont tous cherché, et, je crois, réussi, à traiter de sujets qui l'auraient intéressé.

Michel ARrivé 